\title{
A PROPOSED INFORMATION-BASED MODALITY FOR THE TREATMENT OF CANCER
}

\author{
ANTONY VAN DER MUDE
}

\begin{abstract}
Treatment modalities for cancer involve physical manipulations, such as surgery, immunology, radiation, chemotherapy or gene editing. This is a proposal for an information-based modality. This modality does not change the internal state of the cancer cell directly — instead, the cancer cell is manipulated by giving it information to instruct the cell to perform an action. This modality is based on a theory of Structure Encoding in DNA where information about body part structure controls the epigenetic state of cells in the process of development from pluripotent cells to fully differentiated cells. This implies a model of cancer where cells stuck in an epigenetic state during the process of differentiation lead to malignancy, called the Epigenetic Differentiation Model. A major feature of the Structure Encoding Theory is that the characteristics of the differentiated cell are affected by inter-cellular information passed in the tissue microenvironment, which specifies the exact location of a cell in a body part structure. This is done by exosomes that carry fragments of long non-coding RNA and transposons that convey structure information. In the normal process of epigenetic differentiation, the information passed may lead to apoptosis due to the constraints of a particular body part structure. The proposed treatment involves determining what structure information is being passed in a particular tumor, then adding artificial exosomes that overwhelm the current information with commands for the cells to go into apoptosis.
\end{abstract}

Keywords: cancer; transposons; long non-coding RNA; apoptosis; epigenetics Declarations of Interest: None 


\section{INTRODUCTION}

Treatments for cancer take a variety of forms [1] 2]. These include:

- Manipulation of the physical cancer cell, such as surgery and radiation therapy.

- Manipulation of the processes in the cancer cell, such as antimetabolites, hormone therapy and antitumor antibiotics.

- Manipulation of the mitosis of the cancer cell, such as alkylating agents, topoisomerase inhibitors and mitotic inhibitors.

- Manipulation of the tumor support systems, such as angiogenesis therapy.

- Manipulation of the genes of the cancer cells - gene therapy.

- Manipulation of the immune system - immunotherapy.

The treatment modality I am proposing is based on the manipulation of information passed to the cell, not the manipulation of the cell directly. The idea is to pass to the cancer cells information that will cause them to stop growing or force them into apoptosis. The advantage of using information is that it could be specific for the cancer cells only — that is, the information would not be read by most normal cells, and therefore not affect them.

It is important to identify the distinction between signals and information. A signal is a unit of quantitative data. Morphogens and hormones are examples of intercellular signals. The degree of response to a signal is in proportion to the quantity of a signal. In contrast, the term information refers to some sort of encoded sequence. Information means something as a whole - it is a message - and is less dependent on quantity or concentration. Information is capable of being reduced to a bit string. The DNA code, Morse code, computer text files and music recordings 
are examples of information. In the fields of electrical engineering and computer science, a signal is considered analog, and information is digital.

An information-based modality might seem to be similar to gene therapy [3] [4] [5] since they both involve genetic information, but there are major differences. In this proposed modality, the information being passed is in the form of non-coding RNA or transposons. In contrast, gene therapy for cancer typically involves the delivery of genes using vectors such as viruses to incorporate these genes into the target cell. In this fashion, gene therapy alters the cellular mechanisms of the target cells. The result of gene therapy is a cell with an altered or augmented genome, resulting in different functioning of the cell.

A therapy based on information passing does not change the genetic mechanisms of the cell. Instead, it provides information that instructs a change in the epigenetic behavior, leading to a different phenotype - that is to say, a different behavior of the cell, such as changes to the state of the genetic regulatory network. But the DNA of the cell is not altered. An information-based modality does not have to specifically target cancer cells in the way that gene therapy does. Instead, the information can be tailored in such a way that it is ignored by almost all other cells except the cancer cells and their progenitor.

Since the cells are not being altered through drugs or gene therapy, there is less likelihood that non-cancer cells will be affected. It is expected that intercellular information exchange would only affect cells in a particular epigenetic state, since that state primes the cell to recognize and process the individualized information. Most other cells would ignore the information because it is not targeted to them. This modality, using information rather than signalling, is also more specific than 
therapies that are based on intercellular signal passing. For example, signals such as cytotoxic proteins are also known to affect cells other than the intended target 6].

An information-based modality is based on a theory for Structure Encoding in DNA [7. If this theory is correct, then many cancers are caused by cells getting stuck in the epigenetic process of going from pluripotent stem cells to fully differentiated cells. This results in multiple copies of a single cell at a particular developmental stage that just repeats that stage. The theory states that cells in the process of differentiation will receive information from neighboring cells that identifies where in the body part structure that cell is located. This helps the cell to determine where it is located. This information begins with a rough indicator in the form of morphogen gradients and proceeds to more detailed information in the form of long non-coding RNA.

Given this cancer model, the proposed treatment is to overwhelm the structure information being passed into a cancer cell from its neighbors with information that tells the cell that it should be in the state of apoptosis. Note that instead of disrupting the processes in the cancerous cells, which is done with chemotherapy or gene therapy, the proposed treatment disrupts the information passed between cancerous cells. It is tricking the cancer cell to self-destruct by feeding it the "wrong" information.

This paper is a proposal - it will not specify a treatment in detail. Experimentation will be needed to determine which information sequences are relevant for which cancers, based on the information being passed in the tumor. Then more 
experimentation will be needed to determine what information will be accepted by the tumor and what information leads to apoptosis.

Section 2 will describe the Structure Encoding Theory. Section 3 will present the Epigenetic Differentiation Model of cancer based on this theory. Section 4 contrasts this model with other models. Section 5 will provide evidence for this model. Section 6 will present the hypothesis of an information-based modality for the treatment of cancer in light of this evidence.

\section{Structure Encoding in DNA}

The theory for Structure Encoding in DNA [7] claims that the bulk of the DNA in multicellular organisms encodes for body part structure, not for proteins. This differs from the central dogma of molecular biology that focuses on the translation from DNA to RNA to protein.

It begins with the observation that differentiation in metazoans and plants starts with the morphogenetic processes controlled by chemical signals like morphogens. But as Kerszberg and Wolpert [8] point out: "morphogens may represent a rather crude positional information system, which is then more finely tuned by cell-cell interactions. Clearly, the morphogen gradient does not act alone and is itself specified by a variety of complex cellular mechanisms." That is to say, simple quantitative signals are not sufficient for the precise determination of structure in multicellular organisms. More detailed information passing is also required.

According to this theory, DNA contains structure information in its own encoding in the intergenic regions. This structure data consists of repeated patterns in "non-coding" RNA (in the sense that it does not code for proteins), representing 
different cell types in different locations. This data is manipulated by transposons to determine the fate of each individual cell.

The structure information is organized in a hierarchy. Genes such as the Hox genes control the gross structure, but also control which fine structure details are applicable by selecting the transposons and long non-coding RNAs (lncRNAs). Disabling extraneous structures is accomplished through deactivation by methylation. This process is important in embryogenesis [9]. The overall structure of a body part can be specified at one level, but substructures can be specified once and reused multiple times, like a computer subroutine.

One of the primary purposes of epigenetics is to control the process of going from pluripotent stem cells to fully differentiated cells. This process turns on certain families of genes and turns off others, adjusting the Genetic Regulatory Network to activate the interlocking biochemical processes required for a specialized cell in a complex body part. This requires information to be presented to the cell about its location in the body part structure in the context of the other nearby cells.

Transposons and lncRNA manipulate the DNA [10] 11] [12] [13] [14. They have been found to be active in stem cells in the process of epigenetic differentiation [15]. Their purpose is to fold the DNA in relation to the 3-dimensional structure of the body part the cell is in. To make this possible, it is necessary for structure information to be passed to the cell from its neighbors. Extracellular Vesicles (EVs) such as exosomes [16] pass the information to the cell to determine the structure [17] [18. Studies have shown that lncRNA expression differs depending on the tissue [19] 20. This information is in the form of incomplete fragments of lncRNAs and transposons. The fragment specifies where the particular body part information is 
encoded in the lncRNA or transposon sequence in relation to its neighbors. The fragment tells the cell where exactly it is located in the structure - at the point the fragment ends.

This process occurs in the heterochromatin. LncRNAs have been known to affect chromatin modification [21] 22]. Epigenetic modifications determine the selection of the specific cell type (or apoptosis) at a specific location through transposon splicing and histone modification [23]. This, in turn, configures the genetic regulatory network in the cell.

I shall refer to this theory as the Structure Encoding Theory (SET). I will also use the term Epigenetic Differentiation to refer to the process by which pluripotent stem cells, through multiple generations of cell division, result in fully differentiated cells. This process is driven by epigenetic changes in the cells at different stages, as they react to the signals and information in the microenvironment.

\section{Cancer Arises From Errors in Epigenetic Differentiation}

The theory described in the previous section suggests the cause of many cancers: in the process of epigenetic differentiation, an error either in the genetic information or in the epigenetic state leads to a neoplasm that can become cancerous. The important point is that, regardless of the cause, the cancer cell is stuck in a particular epigenetic state.

Cancer manifests itself in different ways depending on the body part and the stage of epigenetic differentiation where the error arose. What these cancers have in common is that they are stuck in the state where things went wrong. Epigenetic differentiation is a fundamental process in embryogenesis, but it is also part of the 
normal processes of a living organism as it grows, repairs damage, and replaces older cells with new ones. So cancer can arise at any stage of life.

The degree and quality of cancer differ depending on the epigenetic state when it arose. Early in the process, the cell is a stem cell, leading to a cancerous stem cell. Later in the process, the neoplasm manifests itself as an abnormal growth of a particular kind of cell, a situation that is less dangerous than if the error had occurred earlier on in the process. The types of genetic and epigenetic errors that would lead to cancer in the process of epigenetic differentiation may be benign in a fully differentiated cell. These errors would affect that cell and that cell only, but would not lead to an abnormal cancerous growth.

DNA damage appears to be a common cause of cancer. Cancer can be caused by alterations in oncogenes, tumor-suppressor genes, and micro-RNA $(\operatorname{miRNA})[24$. But errors in the processes of epigenetic differentiation, such as methylation errors [9], errors in chromatin, or errors in the regulation of the genetic regulatory network, can also cause cancer [25]. Although cancer is an error of epigenetic differentiation, the root cause can be errors in DNA transcription or errors in the processes of epigenesis.

Some cancers have multiple genetic errors because the correction mechanism is turned off [26]. The theory of Structure Encoding, especially the idea that transposons manipulate the DNA for the purposes of structure determination, can explain why DNA repair genes get deactivated. Repair genes could be turned off in cell differentiation if they interfere with the operation of transposons and lncRNA 27. This would then lead to a sharp increase in the number of genetic errors. 
Some cancers have a purely epigenetic origin. Although this means that these cancer cells would have little or no mutations of any consequence, epigenetic errors result in the abnormal expression of certain genes. An example of this are cancers that are mostly due to incorrect methylation 9 .

As mentioned in the previous section, methylation is important in the process of structure determination. There are two generally recognized problems with methylation in cancer: transcriptional silencing of tumor suppressor genes by $\mathrm{CpG}$ island promoter hypermethylation and global genomic hypomethylation [9] 28] 29]. Hypermethylation often results in disabling genes that repair DNA or trigger apoptosis. Sometimes the effects of methylation silencing and gene mutations have the same effect. Hypomethylation is an error in epigenetic differentiation where the cell has failed to proceed down the path of differentiation to its final state. Instead, the genetic regulatory network for multiple cell types remains active all at the same time, which could lead to cancer [23].

As the process of differentiation continues, intercellular communications establish the microenvironment that is associated with the cells of a particular body part at that stage of development [30. Exosomes are important components and regulators of the cell microenvironment [31] 32]. They have long been recognized as having a part to play in cancer [33 [34 [35] 36] 37] 38, 39] [40. Radiation treatment and cancer have been shown to change the expression of exosomes [41] [42. Exosomes are carriers for a variety of things, such as proteins, RNA and DNA [4]. According to SET, some of these exosomes are carrying detailed structural information in the form of non-coding sequences that are part of transposons and lncRNA [4]. 
Exosomes also carry signals that establish the tissue microenvironment, such as exosomes containing miRNA [45] and morphogens such as Wnt and Hedgehog [46].

The miRNAs that appear in exosomes are used for signaling, which helps to determine the microenvironment 47] 48. For example, exosomes containing miRNA from mesenchymal stem cells have been shown to suppress angiogenesis in breast cancer cells 499. They are also known to control apoptosis [50] [51] [52].

It has been known that lncRNAs control the expression of miRNAs [53] [54] and that the expression of miRNAs is controlled by methylation [55]. There is a strong correlation between the location in the genome of specific miRNAs and HOX genes, indicating their involvement in epigenetic differentiation [56]. The miRNA controls the regulatory network by turning off proteins [57] [58. On the other hand, the p53 repair protein is known to turn on miRNAs [59] 60]. Note that miRNAs are specific for different families of cells since they are involved in differentiation [61].

It could be argued that, since miRNA are small, non-coding RNA sequences, they can be considered information. For example, they are known to bind to messenger RNA ( $m R N A)$ at a site that has been termed a "zipcode" 62. But there is usually no relationship between different miRNAs. Therefore, for the purposes of this paper, they will be considered as individual signals.

Exosomes also carry long non-coding RNA and transposons 63] 64. Some transposons carried by exosomes include certain Long Interspersed Nuclear Elements (LINE-1) 65] 66] and Human Endogenous Retroviruses (HERV) 67]. These contain information sequences in non-coding segments. According to SET, they encode information about where in a body part the cell is located. This is 
especially true if the sequence is incomplete - this indicates the location in the structure.

Note that in this model, the lncRNA and transposons that are passed by exosomes are often specific to that particular cancer, depending on which body part the cancer cell came from. The exception is a case where some common information encoding appears as a subroutine in multiple body parts.

So, given the theory of Structure Encoding in DNA, cancer arises from an error in epigenetic differentiation, caused either by errors in the DNA or by errors in the process of epigenesis. The cancer, though, is dependent on intercellular communications, either through morphogens or through material contained in exosomes.

In summary, the main points are:

- Cancer begins at any step of the process of epigenetic differentiation. The characteristics of the cancer are dependent on the stage of epigenesis.

- Cancer can be caused by genetic errors or by epigenetic errors such as incorrect methylation or intercellular communication.

- Some cancers exhibit a large number of genetic errors because genetic error correction was turned off at the stage where the cancer started.

- Epigenetic differentiation is controlled by signals and information passed in the microenvironment of the cell. Signal passing is done through morphogens and exosomes containing miRNA. Information passing is through exosomes containing lncRNA and transposons. This information also controls the progress of a cancer.

I will refer to this as the Epigenetic Differentiation Model (EDM). In the next section, I will compare this model to other models. 


\section{Epigenetic Differentiation Model of Cancer Compared To Other}

\section{MODELS}

According to EDM, the Cancer Stem Cell $(C S C)$ model 68 69, 60, 71] 72] represents cancers that started in an early stage of differentiation. If a cell becomes cancerous early, then it most likely is or acts like a stem cell. Their ability to form new tissues makes them more likely to lead to the uncontrolled growth of cancer 73] and to metastasize [74.

Although the cancer cells are stuck in a particular stage of epigenetic differentiation, some processes in epigenesis could be continuing. This results in creating a variety of cells derived from the CSC $[75$. This is especially true in the case of cancer stem cells giving rise to cells that are further differentiated, leading to tumor heterogeneity. The epigenetic process will also alter the microenvironment, also affecting other cells [76] [77. Note that this process may not necessarily be one way. Epigenetic processes can sometimes lead to stem cells derived from more differentiated cells [78.

The reason a tumor is not made of just the same stem cell is that the epigenetic process establishes a microenvironment. This can either affect local, non-cancerous cells or turn cancerous cells into non-stem cells. This leads to abnormal structures composed of populations of cells [79].

The CSC model has been contrasted to the older Clonal model, where cancer is the result of multiple clones of a cell with genetic errors [69 [80 [81. The two models are not mutually exclusive [82. It has been suggested that the stem cell model is actually explained by the process of mutation of cancer cells in a clonal model [83]. That cancer can manifest itself in these different ways is explained 
in EDM as a difference in the stage at which the cancer cell got stuck. A cell that becomes cancerous in the early stages of epigenetic differentiation manifest as a cancer stem cell. The clonal model applies later in epigenetic differentiation, going from multipotent to fully defined calls. EDM predicts that cancers can differ because of the cause of the cancer, genetic or epigenetic errors, manifests in different ways depending on the epigenetic stage.

This progress from stem cells to fully differentiated cells has been pointed out by Hanahan and Weinberg: "The origins of CSCs within a solid tumor have not been clarified and indeed may well vary from one tumor type to another. In some tumors, normal tissue stem cells may serve as the cells-of-origin that undergo oncogenic transformation to yield CSCs; in others, partially differentiated transit-amplifying cells, also termed progenitor cells, may suffer the initial oncogenic transformation thereafter assuming more stem-like character." 84

Vogelstein et al. 85] divide mutations into "driver gene" mutations and "passenger mutation". They classify genetic errors in cancer into three core cellular processes: cell fate, cell survival and genome maintenance. In EDM, passenger mutations are the result of the correction mechanism being turned off.

It has been well known that driver mutations are often associated with particular types of cancers [86 [87 [88 [89]. The specificity of driver mutations associated with certain tumors is due to the epigenetic differentiation of the cells, which is implied by EDM. If EDM is correct, the same mutation in a cell of a different type would not lead to cancer, or that some genes are oncogenes in some cell types and tumor suppressors in others [90]. Note that this specificity applies only to driver mutations associated with particular types of cells. There also exist oncogenes that 
are involved in many types of cancers. According to EDM, these genes control the process of epigenetics generally regardless of type.

EDM has features in common with Tissue Organization Field Theory (TOFT) 91] 92. In summary, TOFT is based on the ideas that carcinogens disrupt the interactions that establish the local microenvironment and that proliferation is the default state of all cells. The disruptions of the microenvironment cause the proliferation to disrupt the normal organizational pattern. Both TOFT and EDM place an emphasis on intercellular communications and epigenetics. The main difference is that TOFT focuses on morphogenic fields, but EDM, since it is based on SET, also considers the detailed structure information passed between cells.

As the tumor grows, it requires the materials to help it grow. Facilitated Variation is a theory that explains the development of ancillary components such as angiogenesis 93 . Since the cancer cells are stuck in an intermediate state of epigenetic differentiation, they are predisposed to promoting these features as the tumor grows. This is done by the expression of signals and information in the microenvironment. An example is the promotion of angiogenesis using lncRNAs 94] 95. Cancer Associated Fibroblasts $(C A F)$ and Tumor Associated Macrophages (TAM) are examples of cells that perform these supporting functions. CAFs establish the network of intercellular communication that supports the tumor structure and helps it grow [96]. TAMs, besides establishing intecellular communications in the tumor microenvironment, are also involved in the immune system [97].

Metastasis is often associated with the ability of the cancer to make the epithelialto-mesenchymal transition $(E M T)$ [98, [99, [100. Since EMT is associated with 
early embryogenesis [30, EDM predicts that CSC tumors are more likely to metastasize due to processes like EMT. On the other hand, cancers that start from cells late in the development process, since they are almost fully differentiated, are less likely to metastasize.

How does this model of cancer explain the hallmarks of cancer 101 84? If EDM is correct, most of the hallmarks of cancer are actually properties of epigenetic differentiation that are carried over from the epigenetic state where the cancer arose and do not necessarily require any extra mutations or other modification of the cell beyond this. For example, Schmidt and Chang point out that each of the hallmarks of cancer is modulated by the activity of multiple lncRNAs [102.

Let's address the acquired capability hallmarks one by one.

- Self-Sufficiency in Growth Signals: this is due to the cancer starting in a cell that is in the process of epigenetic differentiation, which is occurring during the natural process of growth.

- Insensitivity to Antigrowth Signals: this is also due to the cell being stuck in a state of growth.

- Evading Apoptosis: in EDM, the ability to evade apoptosis varies depending on the state that the cell was in when the cancer condition arose.

- Limitless Replicative Potential: this is a restatement of the essential point of EDM - the cancer cell is stuck in the process of transition from a pluripotent cell to a fully defined cell. This is part of the growth and development of a body part. Since the process is not turned off, there is limitless replication. 
- Sustained Angiogenesis: angiogenesis is part of the normal process of growth, and is automatically established by the cancer cell.

- Tissue Invasion and Metastasis: in EDM, this, like evasion of apoptosis, varies from tumor to tumor. For example, metastasis is more likely to happen if the cancer cell is capable of EMT. This model predicts that, in general, CSC cancers are more likely to metastasize.

- Reprogramming Energy Metabolism: according to EDM, this is done through the release of signals, such as miRNA, during the process of epigenetic differentiation.

- Evading Immune Destruction: at present, EDM does not address the ability to evade the immune system.

The Hallmarks of Cancer include two enabling characteristics: Genome Instability and Mutation and Tumor-Promoting Inflammation:

- Genome Instability and Mutation: according to EDM, only certain cancers have genome instability, depending on whether or not genome correction was turned off at that stage of differentiation. If it was, the degree of mutation is much higher. But there can be cancers where this does not happen.

- Tumor-Promoting Inflammation: as with the ability to evade immune destruction, the epigenetic model does not address this.

Therefore EDM explains the hallmarks of cancer, except for the immune responses. 


\section{Evidence for the Epigenetic Differentiation Model}

Before discussing the hypothesized treatment, I need to show evidence for the Epigenetic Differentiation Model. This will help determine if a treatment based on this model is feasible.

I claim that cancer is an error in epigenetic differentiation. Some studies have looked into this, for example, showing that the normal repair of a DNA break can occasionally cause heritable silencing of a $\mathrm{CpG}$ island - containing promoter by recruitment of proteins involved in silencing, possibly leading to cancer [103. EDM claims that, although cancer is a problem related to epigenesis, it can occur either with errors in the expression of proteins or with errors in non-coding information that lead to epigenetic errors. Some studies have even shown that mutations in coding regions are lower than in non-coding transcribed regions [104].

EDM claims that DNA repair genes are suppressed during some stages of epigenetics. The tumor suppressor gene p53 is an example of this. At times during the epigenetic differentiation of stem cells, p53 is turned off [105] [106]. Besides genetic errors in p53 that lead to cancer, the p53 genes can be controlled by epigenetic processes involving miRNA [60, transposons [107] and lncRNA 108. For example, knockdown of lncRNA-ST8SIA3 leads to upregulation of genes involved in the p53 response 109. The lncRNA TP73-AS1 is upregulated in cancer - its knockdown inhibits the proliferation of cancer cells [110. Also, field defects can arise due to the supression of repair processes [111].

Exosomes are important in determining the microenvironment and in structure determination. Multiple studies have shown that exosomes are abnormally expressed in cancer [35] [112] 44] 37] [113] [41] [114. 
According to EDM, the closer to the stem cell where the process of epigenesis gets stuck, the more dangerous the cancer. According to SET, this is at the time when the morphogen gradients are being established and morphogenetic genes like the Hox genes are active. These processes are also associated with non-coding RNA, such as HOTAIR, which are expressed near the start of the process of epigenetic differentiation [115]. Consequently, lncRNAs such as HOTAIR are found to be associated with a number of cancers, such as in laryngeal squamous cell carcinoma [44] since they are at the beginning of the process [27] [116].

It has been noted that miRNAs are passed in exosomes and that they influence cell proliferation and apoptosis, both positively and negatively [117 [118, [112. For example, restoration of miR-137 expression resulted in a significant decrease in the proliferation of colorectal cancer [119].

Transposons and lncRNAs appear in the exosomes [120] 121] [122]. They are known to affect the tumor microenvironment [123]. LINE-1 appears in exosomes [124], also HERV retrotransposons [67]. These lncRNAs are implicated in cancer 125] 126] 113. For example, lncRNA-ROR was shown to be increased in malignant hepatocytes [127], and LINE-1 methylation is associated with more aggressive colorectal cancer [128. Some lncRNAs have been observed to regulate apoptosis [129] such as LNC01234 [130, PVT1 [131], GAS5 [129] and URHC [132].

Each tumor cell is different in the expression of lncRNA and transposons in exosomes [133 [112 134 113. Because of this, exosomes are being considered as biomarkers for cancer [135] [44] 64].

It is known that lncRNAs affect the progress of cancer [136 [102 [137. For example, knockdown of lncRNA-ST8SIA3 led to upregulation of genes involved in 
A PROPOSED INFORMATION-BASED MODALITY FOR THE TREATMENT OF CANCER19

the p53 response [109. Other lncRNAs are known to control apoptosis [116] [138, such as C17orf76-AS1 (LRRC75 antisense RNA1) [122, MINCR [139], LINC00460 140, LINC00152 [141], SNHG7 142] and SNHG1 [143]. Lnc-ROR is known to trigger EMT [144] and affects p53 [108.

Studies have shown that methylation errors are as important as genetic errors [28] 29]. Methylation patterns have been shown to differ in different cancers [145]. Methylation errors can go in either direction. Hypermethylation turns off tumor suppressor genes, causing the tumor to grow uncontrollably [146]. Hypomethylation leaves on transposons, such as LINE-1, which is consistent with SET and EDM 147. [128. According to SET, transposons play a part in epigenetic differentiation by determining the location of the cell in a structure. But turning on transposons inappropriately correlates with pathology in certain cancers [148].

EDM and SET predict that structure determination involves the transfer of fragments of lncRNA and transposons, as some studies have shown [135]. But lncRNA fragments may not be identified because of the sampling method. Some methods may autocomplete the fragment or flag a fragment as the whole unit [149] [112.

\section{Hypothesis: An Information-Based Modality for Cancer}

\section{TREATMENT}

The Structure Encoding Theory and the Epigenetic Differentiation Model for cancer suggest a possible modality for cancer treatment.

Presuming that cancer happens when a cell gets stuck in an epigenetic state, then it would be likely that the cells are passing location information using extracellular vesicles such as exosomes between cells to control the epigenetic state. According 
to SET, this would be non-coding RNA and transposons which transfer position information. Also, according to SET, this information will be an initial segment that marks the position. This information will vary from tumor to tumor, depending on the position information related to the cancer cell and its microenvironment.

SET predicts that certain position information passed to a cell could force the cell into apoptosis during normal differentiation. Cells are forced into apoptosis to define a structure, for example, in the creation of voids in tissues or to eliminate cells that are at the edge of a body part. Note that this is different from apoptosis that happens because of DNA transcription errors, for example.

This leads to the statement of the hypothesis:

Hypothesis: An Information-Based Modality for Cancer Treatment

Given Structure Encoding Theory and the Epigenetic Differentiation Model for cancer, many or most cancers pass structure information between the tumor cells in the form of fragments of transposons and lncRNAs. The hypothesis is a treatment for cancer that incorporates into the tumor microenvironment information passed via exosomes to instruct the local cells to go into apoptosis. This will overwhelm the current microenvironment and force the tumor cells to self-destruct.

Using exosomes containing transposons and lncRNA can be done in a variety of ways. For example, HERV affects the immune system, leading to apoptosis [150]. The expression of lncRNA INXS is associated with the expression of BCL-X protein-coding mRNA, leading to apoptosis [151]. This is in contrast to cancer treatments that suppress exosomes 152 .

Another example the case where the loss of the MPP8 gene in the Human Silencing Hub $(H U S H)$ complex reactivated LINE-1, which led to tumor suppression in acute myeloid leukemia $(A M L)$ [153]. Under the EDM model, what is happening 
is that the HUSH complex is turning off the process of epigenetic differentiation. This turns off the activity of the LINE- 1 transposons. Normally, in the process of development of the bone marrow, there are a certain number of hematopoietic stem cells $(H S C)$ that need to be created. This is part of the structure of the marrow and according to SET it is under the control of intercellular information such as the LINE -1 transposons. Once the structure is completed, any further attempt to create more HSCs will be controlled by the action of the LINE-1 in the heterochromatin, resulting in the activation of p21 and p53 mediated apoptosis. Under the EDM model, AML can be due to errors in cancer stem cells including the upregulation of the HUSH complex. If this happens, this cascades down to a problem in the suppression of the LINE- 1 regulation of the bone marrow structure. An information-based modality for the treatment of this cancer would work as follows: instead of attempting to restore the proper functioning of the regulatory network in the cell, either through chemotherapy or gene editing, what is done instead is to flood the site of the leukemia with exosomes containing the particular configuration of the LINE-1 transposon that stops the growth of HSCs in a healthy marrow. It is expected that this would halt the growth of the cancer.

Considerable analysis will be required to determine in general what information is passed in a cancer tumor and also what information is passed during the normal stages of epigenetic differentiation that lead to apoptosis. But once this is determined, flooding the tumor with the desired information may cause it to shrink. Exosomes have been used in various treatments [154]. Research is ongoing to manufacture artificial exosomes [155]. Another method is to have cells naturally generate exosomes then isolate them for use [156] [157. 
Note that studies of transposons and apoptosis in the cell may not help in finding which exosome-containing transposons work best, because apoptosis in those studies is often due to errors in epigenetic development. Therefore, it is important for an information-based modality to determine that the apoptosis is caused by the transfer of information between cells, and not because of errors in the cells themselves that lead to apoptosis.

Of course, this treatment won't work if apoptosis is turned off, which happens, for example, when p53 is disabled. There are two major reasons for apoptosis: the destruction of cells that can't be repaired, and the destruction of cells not required in structure determination. At least one of these methods of apoptosis must be turned on for the treatment to work.

This treatment also presumes that the change can be made by flooding the existing information. For example, increases in exosomes with lncRNA GAS5 has been shown to be associated with apoptosis [158]. Apoptosis is known to start by suppressing lncRNAs [116]. It could be possible to remove information, but this would be harder to do.

The correct treatment in each case could be determined experimentally by trying different lncRNA fragments and seeing what happens. For example, exosomes with different lncRNA sequence lengths are associated with different miRNA expressions 54. It is likely that lncRNA begins with some sort of header information. Presenting the tumor cells with some sequence changes past this header might force it into an apoptosis state. These changes could be substitutions, truncations or extensions.

This leads to the following test of the hypothesis: 


\section{Experimental Test Sequence}

(1) Determine which exosomes containing transposon and lncRNA fragments cause apoptosis during the normal process of cellular differentiation. Create a library of such sequences.

(2) Determine which exosomes containing transposon and lncRNA fragments are associated with which cancer tumors.

(3) Match the transposon and lncRNA fragments in the tumor to the closest fragments that cause apoptosis.

(4) Create artificial exosomes containing the fragments that cause apoptosis.

(5) Flood the tumor with these artificial exosomes and check that this initiates apoptosis.

The first two steps will require the creation of a comprehensive catalog of fragments of transposons and lncRNA and their effects during the process of epigenetic differentiation and cancer. Multiple studies have begun the work of determining how transposons and lncRNA control apoptosis, both in the normal process of development [116, 144, 138, [108, and during cancer [125, 129] [126] 132] 127, 122] 142] 141, 140] 131, 113, 139, 128.

The catalogs will be built from studies that correlate transposons and lncRNA with their associated body part and what fragments lead to what outcome both during normal development and different cancer tumors. It may eventually be possible to develop algorithms that provide general rules for these matches. Finally, some experimentation may be necessary to determine how to deliver the appropriate sequences to the tumor, based on the current sample, and incorporate them into the tumor microenvironment, so that this information overwhelms the current information. 
The challenge of this approach is that it is based on transposons and lncRNAs that are unique to each particular cancer. Research up to now is typically focused on transposons and lncRNAs that show up with many cancers and the ones that are unique to each tumor is ignored. If this information-based modality is to be specific to the tumor, then the unique information being passed between the cells in the tumor will need to be identified.

Information-based treatments are not just limited to inducing apoptosis. The injection of information can also change the epigenetic state, which can ameliorate the effects of cancer. Berdasco and Esteller mention the possibility of reversing the damage to the epigenetic state [25], but their approach is to manipulate the process of epigenetics itself, instead of manipulating the information presented to the cell and letting the cell do it.

A possible advantage of information-based therapy is that it may be less likely for the cancer cells to become resistant to this approach compared to treatments that target signaling pathways. Signaling pathways react to changes in their environment. It is much harder for the cell to epigenetically reprogram itself to react to a change of information.

There is a second modality using morphogens and miRNA signals instead of lncRNA information. This modality is already being considered for treatments. As mentioned before, certain miRNAs act as signals to control apoptosis. Treatments involving miRNA could be very effective [51] [159]. They could be actively manipulated to force the cancer into apoptosis either directly [160] 161 or by removing the methylation of the tumor suppression genes [28]. Also, for cancer cells early in 
the stages of epigenetic differentiation, the concentration of signals associated with morphogenesis can be manipulated.

Since miRNAs often work to suppress gene expression, treatment must be tailored with this in mind. Some treatments work by sponging excess miRNAs 162 163 160. Some treatments involve adding miRNA exosomes [164. Since miRNA is a marker for different cancers because they are specific to different cancers [165, that makes them a good target for a signal-based treatment.

The drawback of using signaling instead of information is that signals themselves are often not specific to the tumor, and could affect non-cancer tissues. Treatment based on information sequences is specific to the tumor and can be identified based on the information already occurring in the exosomes in the tumor microenvironment. Also, signaling is more sensitive to quantity. In contrast, for an information-based treatment, it is only necessary to overwhelm the current information.

\section{Conclusions}

I have suggested a modality for cancer treatment based on controlling the cancer by overwhelming the tumor with information that might cause it to self-destruct. Because information-based treatment does not involve physical manipulation, such as surgery, radiation or chemotherapy, it has advantages. The information can be specially tailored to the tumor, in contrast to a physical manipulation that can possibly have effects beyond the tumor.

There are significant sexual differences in epigenetic differentiation [166] 167] [168] [169]. This may explain the differences in cancer between the sexes and why so many cancers are in the reproductive organs. 
Information-based treatment modalities can be used for other diseases that are related to epigenetic differentiation, for example, schizophrenia, which may be due to structural problems [170. If started early, an information-based treatment could slow or ameliorate the disease.

Information-based treatments could also promote angiogenesis for situations such as heart attacks.

\section{ACKNOWLEDGEMEnts}

I should like to thank Andrew Moore for helpful suggestions that improved the overall presentation and for his encouragement.

\section{REFERENCES}

[1] National Cancer Institute, Types of Cancer Treatment, [Online; accessed 1-July-2020] (2020). URL https: //www.cancer.gov/about-cancer/treatment/types

[2] American Cancer Society, Cancer Treatments, [Online; accessed 1-July-2020] (2020). URL https://www.cancer.org/treatment/treatments-and-side-effects/ treatment-types

[3] C. E. Dunbar, K. A. High, J. K. Joung, D. B. Kohn, K. Ozawa, M. Sadelain, Gene therapy comes of age, Science 359 (6372) (2018).

[4] R. Goswami, G. Subramanian, L. Silayeva, I. Newkirk, D. Doctor, K. Chawla, S. Chattopadhyay, D. Chandra, N. Chilukuri, V. Betapudi, Gene therapy leaves a vicious cycle, Frontiers in oncology 9 (2019) 297.

[5] A. Shahryari, M. Saghaeian Jazi, S. Mohammadi, H. Razavi Nikoo, Z. Nazari, E. S. Hosseini, I. Burtscher, S. J. Mowla, H. Lickert, Development and clinical translation of approved gene therapy products for genetic disorders, Frontiers in genetics 10 (2019) 868.

[6] L.-T. Jia, S.-Y. Chen, A.-G. Yang, Cancer gene therapy targeting cellular apoptosis machinery, Cancer treatment reviews 38 (7) (2012) 868-876. 
[7] A. Van der Mude, Structure encoding in DNA, Journal of Theoretical Biology 492 (2020) 110205.

[8] M. Kerszberg, L. Wolpert, Specifying positional information in the embryo: looking beyond morphogens, Cell 130 (2) (2007) 205-209.

[9] J. G. Herman, S. B. Baylin, Gene silencing in cancer in association with promoter hypermethylation, New England Journal of Medicine 349 (21) (2003) 2042-2054.

[10] M. N. Cabili, C. Trapnell, L. Goff, M. Koziol, B. Tazon-Vega, A. Regev, J. L. Rinn, Integrative annotation of human large intergenic noncoding RNAs reveals global properties and specific subclasses, Genes \& development 25 (18) (2011) 1915-1927.

[11] T. Nagano, P. Fraser, No-nonsense functions for long noncoding RNAs, Cell 145 (2) (2011) $178-181$.

[12] A. Babaian, D. L. Mager, Endogenous retroviral promoter exaptation in human cancer, Mobile DNA 7 (1) (2016) 1-21.

[13] X. Sun, X. Wang, Z. Tang, M. Grivainis, D. Kahler, C. Yun, P. Mita, D. Fenyö, J. D. Boeke, Transcription factor profiling reveals molecular choreography and key regulators of human retrotransposon expression, Proceedings of the National Academy of Sciences 115 (24) (2018) E5526-E5535.

[14] G. J. Faulkner, V. Billon, L1 retrotransposition in the soma: a field jumping ahead, Mobile DNA 9 (1) (2018) 22.

[15] G. G. Schumann, N. V. Fuchs, P. Tristán-Ramos, A. Sebe, Z. Ivics, S. R. Heras, The impact of transposable element activity on therapeutically relevant human stem cells, Mobile DNA 10 (1) (2019) 1-23.

[16] N. P. Hessvik, A. Llorente, Current knowledge on exosome biogenesis and release, Cellular and Molecular Life Sciences 75 (2) (2018) 193-208.

[17] H. Valadi, K. Ekström, A. Bossios, M. Sjöstrand, J. J. Lee, J. O. Lötvall, Exosome-mediated transfer of mRNAs and microRNAs is a novel mechanism of genetic exchange between cells, Nature cell biology 9 (6) (2007) 654-659. 
[18] K. C. Miranda, D. T. Bond, J. Z. Levin, X. Adiconis, A. Sivachenko, C. Russ, D. Brown, C. Nusbaum, L. M. Russo, Massively parallel sequencing of human urinary exosome/microvesicle RNA reveals a predominance of non-coding RNA, PloS one 9 (5) (2014).

[19] M. Sun, W. L. Kraus, Minireview: Long noncoding RNAs: new "links" between gene expression and cellular outcomes in endocrinology, Molecular endocrinology 27 (9) (2013) $1390-1402$

[20] S. J. Liu, T. J. Nowakowski, A. A. Pollen, J. H. Lui, M. A. Horlbeck, F. J. Attenello, D. He, J. S. Weissman, A. R. Kriegstein, A. A. Diaz, et al., Single-cell analysis of long non-coding RNAs in the developing human neocortex, Genome biology 17 (1) (2016) 67.

[21] T. R. Mercer, M. E. Dinger, J. S. Mattick, Long non-coding RNAs: insights into functions, Nature reviews genetics 10 (3) (2009) 155-159.

[22] R. Spizzo, M. I. Almeida, A. Colombatti, G. A. Calin, Long non-coding RNAs and cancer: a new frontier of translational research?, Oncogene 31 (43) (2012) 4577-4587.

[23] F. I. Daniel, K. Cherubini, L. S. Yurgel, M. A. Z. de Figueiredo, F. G. Salum, The role of epigenetic transcription repression and DNA methyltransferases in cancer, Cancer 117 (4) (2011) 677-687.

[24] C. M. Croce, Oncogenes and cancer, New England journal of medicine 358 (5) (2008) 502511.

[25] M. Berdasco, M. Esteller, Aberrant epigenetic landscape in cancer: how cellular identity goes awry, Developmental cell 19 (5) (2010) 698-711.

[26] M. W. Schmitt, M. J. Prindle, L. A. Loeb, Implications of genetic heterogeneity in cancer, Annals of the New York Academy of Sciences 1267 (2012) 110.

[27] M. N. Rossi, F. Antonangeli, LncRNAs: new players in apoptosis control, International journal of cell biology 2014 (2014).

[28] M. Esteller, Aberrant DNA methylation as a cancer-inducing mechanism, Annu. Rev. Pharmacol. Toxicol. 45 (2005) 629-656.

[29] A. Agarwal, R. Polineni, Z. Hussein, I. Vigoda, T. D. Bhagat, S. Bhattacharyya, A. Maitra, A. Verma, Role of epigenetic alterations in the pathogenesis of Barrett's esophagus and 

5 (5) (2012) 382.

[30] M. C. Cabrera, R. E. Hollingsworth, E. M. Hurt, Cancer stem cell plasticity and tumor hierarchy, World journal of stem cells 7 (1) (2015) 27.

[31] F. Wendler, N. Bota-Rabassedas, X. Franch-Marro, Cancer becomes wasteful: Emerging roles of exosomest in cell-fate determination, Journal of extracellular vesicles 2 (1) (2013) 22390 .

[32] M. Colombo, G. Raposo, C. Théry, Biogenesis, secretion, and intercellular interactions of exosomes and other extracellular vesicles, Annual review of cell and developmental biology $30(2014) 255-289$.

[33] M. Silva, S. A Melo, Non-coding RNAs in exosomes: new players in cancer biology, Current genomics 16 (5) (2015) 295-303.

[34] S. Yu, H. Cao, B. Shen, J. Feng, Tumor-derived exosomes in cancer progression and treatment failure, Oncotarget 6 (35) (2015) 37151.

[35] R. Kalluri, The biology and function of exosomes in cancer, The Journal of clinical investigation 126 (4) (2016) 1208-1215.

[36] C. Rajagopal, K. Harikumar, The origin and functions of exosomes in cancer, Frontiers in oncology 8 (2018) 66.

[37] Y.-L. Tai, K.-C. Chen, J.-T. Hsieh, T.-L. Shen, Exosomes in cancer development and clinical applications, Cancer science 109 (8) (2018) 2364-2374.

[38] M. Osaki, F. Okada, Exosomes and their role in cancer progression, Yonago Acta Medica 62 (2) (2019) 182-190.

[39] W. Tian, S. Liu, B. Li, Potential role of exosomes in cancer metastasis, BioMed research international 2019 (2019).

[40] A. Turchinovich, O. Drapkina, A. Tonevitsky, Transcriptome of extracellular vesicles: Stateof-the-art, Frontiers in immunology 10 (2019).

[41] J. Luo, Y. Xiong, P.-f. Fu, E.-c. Li, L. Qu, X. Fan, Z.-j. Cai, A.-f. Lin, Exosomal long noncoding RNAs: biological properties and therapeutic potential in cancer treatment, Journal of Zhejiang University-SCIENCE B 20 (6) (2019) 488-495. 
[42] J. Ni, J. Bucci, D. Malouf, M. Knox, P. Graham, Y. Li, Exosomes in Cancer Radioresistance, Frontiers in oncology 9 (2019) 869.

[43] B. K. Thakur, H. Zhang, A. Becker, I. Matei, Y. Huang, B. Costa-Silva, Y. Zheng, A. Hoshino, H. Brazier, J. Xiang, et al., Double-stranded DNA in exosomes: a novel biomarker in cancer detection, Cell research 24 (6) (2014) 766-769.

[44] M. Dragomir, B. Chen, G. A. Calin, Exosomal lncRNAs as new players in cell-to-cell communication, Translational cancer research 7 (Suppl 2) (2018) S243.

[45] C. Grange, M. Tapparo, F. Collino, L. Vitillo, C. Damasco, M. C. Deregibus, C. Tetta, B. Bussolati, G. Camussi, Microvesicles released from human renal cancer stem cells stimulate angiogenesis and formation of lung premetastatic niche, Cancer research 71 (15) (2011) $5346-5356$.

[46] A. Becker, B. K. Thakur, J. M. Weiss, H. S. Kim, H. Peinado, D. Lyden, Extracellular vesicles in cancer: cell-to-cell mediators of metastasis, Cancer cell 30 (6) (2016) 836-848.

[47] J. Figueroa, L. M. Phillips, T. Shahar, A. Hossain, J. Gumin, H. Kim, A. J. Bean, G. A. Calin, J. Fueyo, E. T. Walters, et al., Exosomes from glioma-associated mesenchymal stem cells increase the tumorigenicity of glioma stem-like cells via transfer of miR-1587, Cancer research 77 (21) (2017) 5808-5819.

[48] A. Kogure, N. Kosaka, T. Ochiya, Cross-talk between cancer cells and their neighbors via miRNA in extracellular vesicles: an emerging player in cancer metastasis, Journal of biomedical science 26 (1) (2019) 1-8.

[49] J.-K. Lee, S.-R. Park, B.-K. Jung, Y.-K. Jeon, Y.-S. Lee, M.-K. Kim, Y.-G. Kim, J.-Y. Jang, C.-W. Kim, Exosomes derived from mesenchymal stem cells suppress angiogenesis by down-regulating VEGF expression in breast cancer cells, PloS one 8 (12) (2013) e84256.

[50] M. Jovanovic, M. Hengartner, miRNAs and apoptosis: RNAs to die for, Oncogene 25 (46) (2006) 6176-6187.

[51] N. Othman, N. H. Nagoor, The role of microRNAs in the regulation of apoptosis in lung cancer and its application in cancer treatment, BioMed research international 2014 (2014). 
A PROPOSED INFORMATION-BASED MODALITY FOR THE TREATMENT OF CANCER31

[52] M. L. Slattery, L. E. Mullany, L. C. Sakoda, R. K. Wolff, W. S. Samowitz, J. S. Herrick, Dysregulated genes and miRNAs in the apoptosis pathway in colorectal cancer patients, Apoptosis $23(3-4)(2018)$ 237-250.

[53] L. Salmena, L. Poliseno, Y. Tay, L. Kats, P. P. Pandolfi, A ceRNA hypothesis: the Rosetta Stone of a hidden RNA language?, Cell 146 (3) (2011) 353-358.

[54] A. Ahadi, S. Brennan, P. J. Kennedy, G. Hutvagner, N. Tran, Long non-coding RNAs harboring miRNA seed regions are enriched in prostate cancer exosomes, Scientific reports 6 (1) (2016) 1-14.

[55] B. Weber, C. Stresemann, B. Brueckner, F. Lyko, Methylation of human microRNA genes in normal and neoplastic cells, Cell cycle 6 (9) (2007) 1001-1005.

[56] G. A. Calin, C. Sevignani, C. D. Dumitru, T. Hyslop, E. Noch, S. Yendamuri, M. Shimizu, S. Rattan, F. Bullrich, M. Negrini, et al., Human microRNA genes are frequently located at fragile sites and genomic regions involved in cancers, Proceedings of the National Academy of Sciences 101 (9) (2004) 2999-3004.

[57] L. P. Lim, N. C. Lau, P. Garrett-Engele, A. Grimson, J. M. Schelter, J. Castle, D. P. Bartel, P. S. Linsley, J. M. Johnson, Microarray analysis shows that some microRNAs downregulate large numbers of target mRNAs, Nature 433 (7027) (2005) 769-773.

[58] A. Esquela-Kerscher, F. J. Slack, Oncomirs-microRNAs with a role in cancer, Nature reviews cancer 6 (4) (2006) 259-269.

[59] G. Wan, R. Mathur, X. Hu, X. Zhang, X. Lu, miRNA response to DNA damage, Trends in biochemical sciences 36 (9) (2011) 478-484.

[60] H. Hu, R. A. Gatti, MicroRNAs: new players in the DNA damage response, Journal of molecular cell biology 3 (3) (2011) 151-158.

[61] M. Negrini, M. Ferracin, S. Sabbioni, C. M. Croce, MicroRNAs in human cancer: from research to therapy, Journal of cell science 120 (11) (2007) 1833-1840.

[62] M. F. Bolukbasi, A. Mizrak, G. B. Ozdener, S. Madlener, T. Ströbel, E. P. Erkan, J.-B. Fan, X. O. Breakefield, O. Saydam, miR-1289 and "Zipcode"-like sequence enrich mRNAs in microvesicles, Molecular Therapy-Nucleic Acids 1 (2012) e10. 
[63] S. Yang, X. Li, Recent advances in extracellular vesicles enriched with non-coding RNAs related to cancers, Genes \& diseases 5 (1) (2018) 36-42.

[64] S. A. Hinger, D. J. Cha, J. L. Franklin, J. N. Higginbotham, Y. Dou, J. Ping, L. Shu, N. Prasad, S. Levy, B. Zhang, et al., Diverse long RNAs are differentially sorted into extracellular vesicles secreted by colorectal cancer cells, Cell reports 25 (3) (2018) 715-725.

[65] C. Spadafora, A LINE-1-encoded reverse transcriptase-dependent regulatory mechanism is active in embryogenesis and tumorigenesis, Ann NY Acad Sci 1341 (1) (2015) 164-171.

[66] D. Ardeljan, M. S. Taylor, D. T. Ting, K. H. Burns, The human long interspersed element1 retrotransposon: an emerging biomarker of neoplasia, Clinical chemistry 63 (4) (2017) $816-822$.

[67] L. Balaj, R. Lessard, L. Dai, Y.-J. Cho, S. L. Pomeroy, X. O. Breakefield, J. Skog, Tumour microvesicles contain retrotransposon elements and amplified oncogene sequences, Nature communications 2 (1) (2011) 1-9.

[68] J. E. Visvader, G. J. Lindeman, Cancer stem cells in solid tumours: accumulating evidence and unresolved questions, Nature reviews cancer 8 (10) (2008) 755-768.

[69] M. Shackleton, E. Quintana, E. R. Fearon, S. J. Morrison, Heterogeneity in cancer: cancer stem cells versus clonal evolution, Cell 138 (5) (2009) 822-829.

[70] P. B. Gupta, C. L. Chaffer, R. A. Weinberg, Cancer stem cells: mirage or reality?, Nature medicine 15 (9) (2009) 1010-1012.

[71] M. López-Lázaro, Stem cell division theory of cancer, Cell Cycle 14 (16) (2015) 2547.

[72] G. Manzo, Similarities between embryo development and cancer process suggest new strategies for research and therapy of tumors: a new point of view, Frontiers in cell and developmental biology 7 (2019) 20.

[73] C. Tomasetti, B. Vogelstein, Variation in cancer risk among tissues can be explained by the number of stem cell divisions, Science 347 (6217) (2015) 78-81.

[74] M. López-Lázaro, The migration ability of stem cells can explain the existence of cancer of unknown primary site. Rethinking metastasis, Oncoscience 2 (5) (2015) 467.

[75] S. J. Sundar, J. K. Hsieh, S. Manjila, J. D. Lathia, A. Sloan, The role of cancer stem cells in glioblastoma, Neurosurgical focus 37 (6) (2014) E6. 
[76] D. Hanahan, L. M. Coussens, Accessories to the crime: functions of cells recruited to the tumor microenvironment, Cancer cell 21 (3) (2012) 309-322.

[77] A. Kreso, J. E. Dick, Evolution of the cancer stem cell model, Cell stem cell 14 (3) (2014) $275-291$.

[78] N. D. Marjanovic, R. A. Weinberg, C. L. Chaffer, Cell plasticity and heterogeneity in cancer, Clinical chemistry 59 (1) (2013) 168-179.

[79] P. Dalerba, R. W. Cho, M. F. Clarke, Cancer stem cells: models and concepts, Annu. Rev. Med. 58 (2007) 267-284

[80] W. Wang, Y. Quan, Q. Fu, Y. Liu, Y. Liang, J. Wu, G. Yang, C. Luo, Q. Ouyang, Y. Wang, Dynamics between cancer cell subpopulations reveals a model coordinating with both hierarchical and stochastic concepts, PloS one 9 (1) (2014).

[81] M. Greaves, C. C. Maley, Clonal evolution in cancer, Nature 481 (7381) (2012) 306-313.

[82] J. N. Rich, Cancer stem cells: understanding tumor hierarchy and heterogeneity, Medicine 95 (Suppl 1) (2016).

[83] G. van Niekerk, L. M. Davids, S. M. Hattingh, A.-M. Engelbrecht, Cancer stem cells: A product of clonal evolution?, International Journal of Cancer 140 (5) (2017) 993-999.

[84] D. Hanahan, R. A. Weinberg, Hallmarks of cancer: the next generation, cell 144 (5) (2011) 646-674.

[85] B. Vogelstein, N. Papadopoulos, V. E. Velculescu, S. Zhou, L. A. Diaz, K. W. Kinzler, Cancer genome landscapes, science 339 (6127) (2013) 1546-1558.

[86] L. D. Wood, D. W. Parsons, S. Jones, J. Lin, T. Sjöblom, R. J. Leary, D. Shen, S. M. Boca, T. Barber, J. Ptak, et al., The genomic landscapes of human breast and colorectal cancers, Science 318 (5853) (2007) 1108-1113.

[87] F. Vandin, E. Upfal, B. J. Raphael, De novo discovery of mutated driver pathways in cancer, Genome research 22 (2) (2012) 375-385.

[88] D. M. Roy, L. A. Walsh, T. A. Chan, Driver mutations of cancer epigenomes, Protein \& cell 5 (4) (2014) 265-296. 
[89] J. Iranzo, I. Martincorena, E. V. Koonin, Cancer-mutation network and the number and specificity of driver mutations, Proceedings of the National Academy of Sciences 115 (26) (2018) E6010-E6019

[90] A. Colaprico, C. Olsen, M. H. Bailey, G. J. Odom, T. Terkelsen, T. C. Silva, A. V. Olsen, L. Cantini, A. Zinovyev, E. Barillot, et al., Interpreting pathways to discover cancer driver genes with moonlight, Nature communications 11 (1) (2020) 1-17.

[91] A. M. Soto, C. Sonnenschein, The somatic mutation theory of cancer: growing problems with the paradigm?, BioEssays 26 (10) (2004) 1097-1107.

[92] S. G. Baker, A cancer theory kerfuffle can lead to new lines of research, Journal of the National Cancer Institute 107 (2) (2015) dju405.

[93] J. Gerhart, M. Kirschner, The theory of facilitated variation, Proceedings of the National Academy of Sciences 104 (suppl 1) (2007) 8582-8589.

[94] H.-L. Lang, G.-W. Hu, B. Zhang, W. Kuang, Y. Chen, L. Wu, G.-H. Xu, Glioma cells enhance angiogenesis and inhibit endothelial cell apoptosis through the release of exosomes that contain long non-coding RNA CCAT2, Oncology reports 38 (2) (2017) 785-798.

[95] H. Lang, G. Hu, Y. Chen, Y. Liu, W. Tu, Y. Lu, L. Wu, G. Xu, Glioma cells promote angiogenesis through the release of exosomes containing long non-coding RNA POU3F3, Eur Rev Med Pharmacol Sci 21 (5) (2017) 959-972.

[96] T.-X. Huang, X.-Y. Guan, L. Fu, Therapeutic targeting of the crosstalk between cancerassociated fibroblasts and cancer stem cells, American journal of cancer research 9 (9) (2019) 1889.

[97] M. Jinushi, S. Chiba, H. Yoshiyama, K. Masutomi, I. Kinoshita, H. Dosaka-Akita, H. Yagita, A. Takaoka, H. Tahara, Tumor-associated macrophages regulate tumorigenicity and anticancer drug responses of cancer stem/initiating cells, Proceedings of the National Academy of Sciences 108 (30) (2011) 12425-12430.

[98] J. P. Thiery, H. Acloque, R. Y. Huang, M. A. Nieto, Epithelial-mesenchymal transitions in development and disease, cell 139 (5) (2009) 871-890.

[99] D. Kong, Y. Li, Z. Wang, F. H. Sarkar, Cancer stem cells and epithelial-to-mesenchymal transition (EMT)-phenotypic cells: are they cousins or twins?, Cancers 3 (1) (2011) 716-729. 
[100] M. K. Jolly, M. Boareto, B. Huang, D. Jia, M. Lu, E. Ben-Jacob, J. N. Onuchic, H. Levine, Implications of the hybrid epithelial/mesenchymal phenotype in metastasis, Frontiers in oncology 5 (2015) 155.

[101] D. Hanahan, R. A. Weinberg, The hallmarks of cancer, cell 100 (1) (2000) 57-70.

[102] A. M. Schmitt, H. Y. Chang, Long noncoding RNAs in cancer pathways, Cancer cell 29 (4) (2016) 452-463.

[103] H. M. O’Hagan, H. P. Mohammad, S. B. Baylin, Double strand breaks can initiate gene silencing and SIRT1-dependent onset of DNA methylation in an exogenous promoter CpG island, PLoS genetics 4 (8) (2008).

[104] M. Tuna, C. I. Amos, Genomic sequencing in cancer, Cancer letters 340 (2) (2013) 161-170.

[105] E. Soto-Reyes, F. Recillas-Targa, Epigenetic regulation of the human p53 gene promoter by the CTCF transcription factor in transformed cell lines, Oncogene 29 (15) (2010) 2217-2227.

[106] R. Aloni-Grinstein, Y. Shetzer, T. Kaufman, V. Rotter, p53: the barrier to cancer stem cell formation, FEBS letters 588 (16) (2014) 2580-2589.

[107] R. Rebollo, M. T. Romanish, D. L. Mager, Transposable elements: an abundant and natural source of regulatory sequences for host genes, Annual review of genetics 46 (2012) 21-42.

[108] E. A. Toraih, A. El-Wazir, M. H. Hussein, M. S. Khashana, A. Matter, M. S. Fawzy, S. Hosny, Expression of long intergenic non-coding RNA, regulator of reprogramming, and its prognostic value in patients with glioblastoma, The International journal of biological markers 34 (1) (2019) 69-79.

[109] S. Loewer, M. N. Cabili, M. Guttman, Y.-H. Loh, K. Thomas, I. H. Park, M. Garber, M. Curran, T. Onder, S. Agarwal, et al., Large intergenic non-coding RNA-RoR modulates reprogramming of human induced pluripotent stem cells, Nature genetics 42 (12) (2010) 1113.

[110] X. Li, X. Wang, L. Mao, S. Zhao, H. Wei, LncRNA TP73-AS1 predicts poor prognosis and promotes cell proliferation in ovarian cancer via cell cycle and apoptosis regulation, Molecular medicine reports 18 (1) (2018) 516-522. 
[111] A. Facista, H. Nguyen, C. Lewis, A. R. Prasad, L. Ramsey, B. Zaitlin, V. Nfonsam, R. S. Krouse, H. Bernstein, C. M. Payne, et al., Deficient expression of DNA repair enzymes in early progression to sporadic colon cancer, Genome integrity 3 (1) (2012) 3.

[112] Z. Wei, A. O. Batagov, S. Schinelli, J. Wang, Y. Wang, R. El Fatimy, R. Rabinovsky, L. Balaj, C. C. Chen, F. Hochberg, et al., Coding and noncoding landscape of extracellular RNA released by human glioma stem cells, Nature communications 8 (1) (2017) 1-15.

[113] W. Zhao, Y. Liu, C. Zhang, C. Duan, Multiple Roles of Exosomal Long Noncoding RNAs in Cancers, BioMed research international 2019 (2019).

[114] F. Lucien, H. S. Leong, The role of extracellular vesicles in cancer microenvironment and metastasis: myths and challenges, Biochemical Society Transactions 47 (1) (2019) 273-280.

[115] X.-h. Shen, P. Qi, X. Du, Long non-coding RNAs in cancer invasion and metastasis, Modern Pathology 28 (1) (2015) 4-13.

[116] J. Zhao, Y. Liu, W. Zhang, Z. Zhou, J. Wu, P. Cui, Y. Zhang, G. Huang, Long noncoding RNA Linc00152 is involved in cell cycle arrest, apoptosis, epithelial to mesenchymal transition, cell migration and invasion in gastric cancer, Cell cycle 14 (19) (2015) 3112-3123.

[117] J. Godlewski, M. O. Nowicki, A. Bronisz, S. Williams, A. Otsuki, G. Nuovo, A. RayChaudhury, H. B. Newton, E. A. Chiocca, S. Lawler, Targeting of the Bmi-1 oncogene/stem cell renewal factor by microRNA-128 inhibits glioma proliferation and self-renewal, Cancer research 68 (22) (2008) 9125-9130.

[118] C. L. A. Yeung, N.-N. Co, T. Tsuruga, T.-L. Yeung, S.-Y. Kwan, C. S. Leung, Y. Li, E. S. Lu, K. Kwan, K.-K. Wong, et al., Exosomal transfer of stroma-derived miR21 confers paclitaxel resistance in ovarian cancer cells through targeting APAF1, Nature communications 7 (1) (2016) $1-14$.

[119] F. Balaguer, A. Link, J. J. Lozano, M. Cuatrecasas, T. Nagasaka, C. R. Boland, A. Goel, Epigenetic silencing of miR-137 is an early event in colorectal carcinogenesis, Cancer research 70 (16) (2010) 6609-6618.

[120] U. Gezer, E. Özgür, M. Cetinkaya, M. Isin, N. Dalay, Long non-coding RNAs with low expression levels in cells are enriched in secreted exosomes, Cell biology international 38 (9) (2014) 1076-1079. 


\section{A PROPOSED INFORMATION-BASED MODALITY FOR THE TREATMENT OF CANCER37}

[121] C. Kahlert, S. A. Melo, A. Protopopov, J. Tang, S. Seth, M. Koch, J. Zhang, J. Weitz, L. Chin, A. Futreal, et al., Identification of double-stranded genomic DNA spanning all chromosomes with mutated KRAS and p53 DNA in the serum exosomes of patients with pancreatic cancer, Journal of Biological Chemistry 289 (7) (2014) 3869-3875.

[122] M. Chen, R. Xu, H. Ji, D. W. Greening, A. Rai, K. Izumikawa, H. Ishikawa, N. Takahashi, R. J. Simpson, Transcriptome and long noncoding RNA sequencing of three extracellular vesicle subtypes released from the human colon cancer LIM1863 cell line, Scientific reports 6 (2016) 38397.

[123] S. Wang, X. Li, R. Zhu, Q. Han, R. C. Zhao, Lung cancer exosomes initiate global long noncoding RNA changes in mesenchymal stem cells, International journal of oncology 48 (2) (2016) 681-689.

[124] E. N. Nolte-'t Hoen, H. P. Buermans, M. Waasdorp, W. Stoorvogel, M. H. Wauben, P. A. 't Hoen, Deep sequencing of RNA from immune cell-derived vesicles uncovers the selective incorporation of small non-coding RNA biotypes with potential regulatory functions, Nucleic acids research 40 (18) (2012) 9272-9285.

[125] E. A. Gibb, C. J. Brown, W. L. Lam, The functional role of long non-coding RNA in human carcinomas, Molecular cancer 10 (1) (2011) 38.

[126] P. E. Carreira, S. R. Richardson, G. J. Faulkner, L1 retrotransposons, cancer stem cells and oncogenesis, The FEBS journal 281 (1) (2014) 63-73

[127] K. Takahashi, I. K. Yan, T. Kogure, H. Haga, T. Patel, Extracellular vesicle-mediated transfer of long non-coding RNA ROR modulates chemosensitivity in human hepatocellular cancer, FEBS open Bio 4 (1) (2014) 458-467.

[128] A.-D. Voichitoiu, B. M. Radu, L. Pavelescu, D. Cretoiu, A. T. Deftu, N. Suciu, S. M. Cretoiu, Extracellular Vesicles in Cancer, in: Extracellular Vesicles, IntechOpen, 2019.

[129] M. Pickard, M. Mourtada-Maarabouni, G. Williams, Long non-coding RNA GAS5 regulates apoptosis in prostate cancer cell lines, Biochimica et Biophysica Acta (BBA)-Molecular Basis of Disease 1832 (10) (2013) 1613-1623. 
[130] M. Ghaffar, S. Khodahemmati, J. Li, M. Shahzad, M. Wang, Y. Wang, C. Li, S. Chen, Y. Zeng, Long non-coding RNA LINC01234 regulates proliferation, invasion and apoptosis in esophageal cancer cells, Journal of Cancer 9 (22) (2018) 4242.

[131] M. Salehi, M. Sharifi, Induction of apoptosis and necrosis in human acute erythroleukemia cells by inhibition of long non-coding RNA PVT1, Molecular biology research communications 7 (2) (2018) 89.

[132] W.-H. Xu, J.-B. Zhang, Z. Dang, X. Li, T. Zhou, J. Liu, D.-S. Wang, W.-J. Song, K.-F. Dou, Long non-coding RNA URHC regulates cell proliferation and apoptosis via ZAK through the ERK/MAPK signaling pathway in hepatocellular carcinoma, International journal of biological sciences 10 (7) (2014) 664 .

[133] C. C. Li, S. A. Eaton, P. E. Young, M. Lee, R. Shuttleworth, D. T. Humphreys, G. E. Grau, V. Combes, M. Bebawy, J. Gong, et al., Glioma microvesicles carry selectively packaged coding and non-coding RNAs which alter gene expression in recipient cells, RNA biology $10(8)(2013)$ 1333-1344.

[134] X. Yan, Z. Hu, Y. Feng, X. Hu, J. Yuan, S. D. Zhao, Y. Zhang, L. Yang, W. Shan, Q. He, et al., Comprehensive genomic characterization of long non-coding RNAs across human cancers, Cancer cell 28 (4) (2015) 529-540.

[135] D. Ke, H. Li, Y. Zhang, Y. An, H. Fu, X. Fang, X. Zheng, The combination of circulating long noncoding RNAs AK001058, INHBA-AS1, MIR4435-2HG, and CEBPA-AS1 fragments in plasma serve as diagnostic markers for gastric cancer, Oncotarget 8 (13) (2017) 21516.

[136] C. Hewson, D. Capraro, J. Burdach, N. Whitaker, K. V. Morris, Extracellular vesicle associated long non-coding RNAs functionally enhance cell viability, Non-coding RNA research $1(1)(2016) 3-11$.

[137] Z. Sun, S. Yang, Q. Zhou, G. Wang, J. Song, Z. Li, Z. Zhang, J. Xu, K. Xia, Y. Chang, et al., Emerging role of exosome-derived long non-coding RNAs in tumor microenvironment, Molecular cancer 17 (1) (2018) 82.

[138] L. Han, E. W.-F. Lam, Y. Sun, Extracellular vesicles in the tumor microenvironment: old stories, but new tales, Molecular cancer 18 (1) (2019) 59. 
A PROPOSED INFORMATION-BASED MODALITY FOR THE TREATMENT OF CANCER39

[139] S. Chen, T. Gu, Z. Lu, L. Qiu, G. Xiao, X. Zhu, F. Li, H. Yu, G. Li, H. Liu, Roles of MYCtargeting long non-coding RNA MINCR in cell cycle regulation and apoptosis in non-small cell lung Cancer, Respiratory research 20 (1) (2019) 1-11.

[140] Y. Lian, C. Yan, H. Xu, J. Yang, Y. Yu, J. Zhou, Y. Shi, J. Ren, G. Ji, K. Wang, A novel lncRNA, LINC00460, affects cell proliferation and apoptosis by regulating KLF2 and CUL4A expression in colorectal cancer, Molecular Therapy-Nucleic Acids 12 (2018) 684-697.

[141] Z. Bian, J. Zhang, M. Li, Y. Feng, S. Yao, M. Song, X. Qi, B. Fei, Y. Yin, D. Hua, et al., Long non-coding RNA LINC00152 promotes cell proliferation, metastasis, and confers 5-FU resistance in colorectal cancer by inhibiting miR-139-5p, Oncogenesis 6 (11) (2017) 1-11.

[142] M. Wang, J. Liu, Q. Liu, Q. Xu, T. Li, S. Jin, T. Xia, LncRNA SNHG7 promotes the proliferation and inhibits apoptosis of gastric cancer cells by repressing the P15 and P16 expression, Eur Rev Med Pharmacol Sci 21 (20) (2017) 4613-4622.

[143] N. Zhang, X. Meng, L. Mei, J. Hu, C. Zhao, W. Chen, The long non-coding RNA SNHG1 attenuates cell apoptosis by regulating miR-195 and BCL2-like protein 2 in human cardiomyocytes, Cellular Physiology and Biochemistry 50 (3) (2018) 1029-1040.

[144] Y. Lou, H. Jiang, Z. Cui, L. Wang, X. Wang, T. Tian, Linc-ROR induces epithelial-tomesenchymal transition in ovarian cancer by increasing Wnt/ $\beta$-catenin signaling, Oncotarget 8 (41) (2017) 69983.

[145] A. F. Fernandez, Y. Assenov, J. I. Martin-Subero, B. Balint, R. Siebert, H. Taniguchi, H. Yamamoto, M. Hidalgo, A.-C. Tan, O. Galm, et al., A DNA methylation fingerprint of 1628 human samples, Genome research 22 (2) (2012) 407-419.

[146] H. Miao, L. Wang, H. Zhan, J. Dai, Y. Chang, F. Wu, T. Liu, Z. Liu, C. Gao, L. Li, et al., A long noncoding RNA distributed in both nucleus and cytoplasm operates in the PYCARD-regulated apoptosis by coordinating the epigenetic and translational regulation, PLoS genetics 15 (5) (2019) e1008144.

[147] K. Hur, P. Cejas, J. Feliu, J. Moreno-Rubio, E. Burgos, C. R. Boland, A. Goel, Hypomethylation of long interspersed nuclear element-1 (LINE-1) leads to activation of proto-oncogenes in human colorectal cancer metastasis, Gut 63 (4) (2014) 635-646. 
[148] E. Sunami, M. De Maat, A. Vu, R. R. Turner, D. S. Hoon, LINE-1 hypomethylation during primary colon cancer progression, PloS one 6 (4) (2011).

[149] X. Huang, T. Yuan, M. Tschannen, Z. Sun, H. Jacob, M. Du, M. Liang, R. L. Dittmar, Y. Liu, M. Liang, et al., Characterization of human plasma-derived exosomal RNAs by deep sequencing, BMC genomics 14 (1) (2013) 319.

[150] N. Bannert, H. Hofmann, A. Block, O. Hohn, HERVs new role in cancer: from accused perpetrators to cheerful protectors, Frontiers in microbiology 9 (2018) 178.

[151] C. DeOcesano-Pereira, M. S. Amaral, K. S. Parreira, A. C. Ayupe, J. F. Jacysyn, G. P. Amarante-Mendes, E. M. Reis, S. Verjovski-Almeida, Long non-coding RNA INXS is a critical mediator of BCL-XS induced apoptosis, Nucleic acids research 42 (13) (2014) 83438355.

[152] A. M. Marleau, C.-S. Chen, J. A. Joyce, R. H. Tullis, Exosome removal as a therapeutic adjuvant in cancer, Journal of translational medicine 10 (1) (2012) 1-12.

[153] Z. Gu, Y. Liu, Y. Zhang, H. Cao, J. Lyu, X. Wang, A. Wylie, S. J. Newkirk, A. E. Jones, M. Lee, et al., Silencing of line-1 retrotransposons is a selective dependency of myeloid leukemia, Nature genetics (2021) 1-11.

[154] T. Lener, M. Gimona, L. Aigner, V. Börger, E. Buzas, G. Camussi, N. Chaput, D. Chatterjee, F. A. Court, H. A. d. Portillo, et al., Applying extracellular vesicles based therapeutics in clinical trials-an ISEV position paper, Journal of extracellular vesicles 4 (1) (2015) 30087.

[155] P. García-Manrique, M. Matos, G. Gutiérrez, C. Pazos, M. C. Blanco-López, Therapeutic biomaterials based on extracellular vesicles: classification of bio-engineering and mimetic preparation routes, Journal of extracellular vesicles 7 (1) (2018) 1422676.

[156] S.-i. Ohno, M. Takanashi, K. Sudo, S. Ueda, A. Ishikawa, N. Matsuyama, K. Fujita, T. Mizutani, T. Ohgi, T. Ochiya, et al., Systemically injected exosomes targeted to EGFR deliver antitumor microRNA to breast cancer cells, Molecular Therapy 21 (1) (2013) 185-191.

[157] H. Zhang, Y. Wang, M. Bai, J. Wang, K. Zhu, R. Liu, S. Ge, J. Li, T. Ning, T. Deng, et al., Exosomes serve as nanoparticles to suppress tumor growth and angiogenesis in gastric cancer by delivering hepatocyte growth factor si RNA, Cancer science 109 (3) (2018) 629-641. 
[158] O. Koldemir, E. Özgür, U. Gezer, Accumulation of GAS5 in exosomes is a marker of apoptosis induction, Biomedical Reports 6 (3) (2017) 358-362.

[159] S. I. Rothschild, microRNA therapies in cancer, Molecular and cellular therapies 2 (1) (2014) 7.

[160] V. Pileczki, R. Cojocneanu-Petric, M. Maralani, I. B. Neagoe, R. Sandulescu, MicroRNAs as regulators of apoptosis mechanisms in cancer, Clujul Medical 89 (1) (2016) 50.

[161] B. Mansoori, A. Mohammadi, S. Shirjang, E. Baghbani, Micro RNA 34a and Let-7a expression in human breast cancers is associated with apoptotic expression genes, Asian Pacific Journal of Cancer Prevention 17 (4) (2016) 1887-1890.

[162] M. S. Ebert, J. R. Neilson, P. A. Sharp, MicroRNA sponges: competitive inhibitors of small RNAs in mammalian cells, Nature methods 4 (9) (2007) 721-726.

[163] M. S. Ebert, P. A. Sharp, MicroRNA sponges: progress and possibilities, Rna 16 (11) (2010) $2043-2050$

[164] A. G. Bader, D. Brown, M. Winkler, The promise of microRNA replacement therapy, Cancer research 70 (18) (2010) 7027-7030.

[165] J. Hayes, P. P. Peruzzi, S. Lawler, MicroRNAs in cancer: biomarkers, functions and therapy, Trends in molecular medicine 20 (8) (2014) 460-469.

[166] J. McDonald, M. Matzke, A. Matzke, Host defenses to transposable elements and the evolution of genomic imprinting, Cytogenetic and genome research 110 (1-4) (2005) 242-249.

[167] A. J. Pask, A. T. Papenfuss, E. I. Ager, K. A. McColl, T. P. Speed, M. B. Renfree, Analysis of the platypus genome suggests a transposon origin for mammalian imprinting, Genome biology 10 (1) (2009) R1.

[168] A. C. Ferguson-Smith, Genomic imprinting: the emergence of an epigenetic paradigm, Nature Reviews Genetics 12 (8) (2011) 565

[169] J. Autuoro, S. Pirnie, G. Carmichael, Long noncoding RNAs in imprinting and X chromosome inactivation, Biomolecules 4 (1) (2014) 76-100.

[170] P. V. Gejman, A. R. Sanders, J. Duan, The role of genetics in the etiology of schizophrenia, Psychiatric Clinics 33 (1) (2010) 35-66. 
32 Second Avenue \#332, Burlington MA, 01803

Email address: vandermude@acm.org 